\title{
Métodos para homogenizar y rellenar datos de viento de la torre meteorológica del Parque Eólico Villonaco en Loja-Ecuador
}

\author{
Diego Jijón ${ }^{1 *}$, Jessica Constante ${ }^{1}$, Marcelo Moya ${ }^{1}$, Gonzalo Guerrón ${ }^{1}$ \\ ${ }^{1}$ Instituto Nacional de Eficiencia Energética y Energías Renovables, Av. 6 de diciembre N33-32 e Ignacio Bossano, Quito, Ecuador. \\ *Autor principal/Corresponding author, e-mail: juan.jijon@iner.gob.ec \\ Editado por/Edited by: Cesar Zambrano, Ph.D. \\ Recibido/Received: 2015/02/06. Aceptado/Accepted: 2015/03/30. \\ Publicado en línea/Published on Web: 2015/12/30. Impreso/Printed: 2015/12/30.
}

\section{Methods to homogenize and fill the wind data from the meteorological tower of Villonaco Wind Farm in Loja-Ecuador}

\begin{abstract}
A new methodology of wind data processing used in feasibility and optimization of wind farms is presented in this work. The wind data of the meteorological tower provided by CELEC-EP is analyzed through a quality control of suspicious values, according to wind speeds greater than $50(\mathrm{~m} / \mathrm{s})$ and a daily statistical relationship proposed in this work for the wind measurements at 20, 40 and 60 meters high. The suspicious values are processed and filled across of the statistical relationship among the different wind speed data at the three heights of measurement as a multivariate function. On the other hand, the turbulence intensity behaves as an exponential decay function of the average and standard deviation wind speed data. This work analyzes two methodologies for missing wind speed data filled, both were compared and one is chosen for wind data processing. The wind speed data quality control and homogenization were automatized since the supervisory control and data acquisition system "SCADA" of the Villonaco Wind Farm.
\end{abstract}

Keywords. Wind data analysis, wind database, wind data processing, quality control and homogenization.

\section{Resumen}

En este trabajo se presenta una nueva metodología para procesar los datos de viento que son usados en los análisis de pre-factibilidad y optimización de parques eólicos. Los datos de la torre meteorológica de Villonaco, entregados por CELEC-EP, fueron analizados a través de un control de calidad que consistió en reconocer los valores dudosos de velocidades de viento, medidas a 20,40 y 60 metros de altura, mayores a $50(\mathrm{~m} / \mathrm{s})$ y los cuales no corresponden a una relación estadística diaria propuesta en este estudio. Los valores dudosos son rellenados a través de una comparación estadística entre las mediciones de velocidad de viento, y así, se logró encontrar una relación entre las mediciones para una altura, en relación de las otras como una función multivariante. Por otro lado, la intensidad de turbulencia se comporta como una función de decaimiento exponencial entre la velocidad media y desviación estándar de velocidad del viento. Se establecieron dos metodologías de relleno de datos, las cuales fueron comparadas al final de este trabajo. El análisis fue automatizado desde los archivos planos recopilados por el sistema de control y adquisición de datos "SCADA" del Parque Eólico Villonaco.

Palabras Clave. Análisis de viento, bases de datos de viento, control de calidad y homogenización, procesamiento de datos de viento.

\section{Introducción}

La torre meteorológica de Villonaco se encuentra en la provincia de Loja a 4 kilómetros de la capital con coordenadas $79^{\circ} 15^{\prime} 35.16^{\prime \prime}$ al oeste (Longitud) y $3^{\circ} 59^{\prime} 49.58^{\prime}$ al sur (Latitud), instalada a una altura aproximada de 2720 metros sobre el nivel del mar, la cual permite estudiar el comportamiento del viento en el cerro de Villonaco para los estudios de potencia y energía generados por el parque eólico [1].

El Parque Eólico Villonaco posee una potencia nominal de 16.5 MW suministrada por 11 generadores tipo GoldWind 70/1500, el cual se espera que genere aproximadamente $60 \mathrm{GWh}$ al año [1, 2]. La torre meteorológica de Villonaco registra los datos de dirección del viento a 38 y 60 metros de altura, y para la velocidad de viento lo hace a 20, 40 y 60 metros de altura. Esta estación 


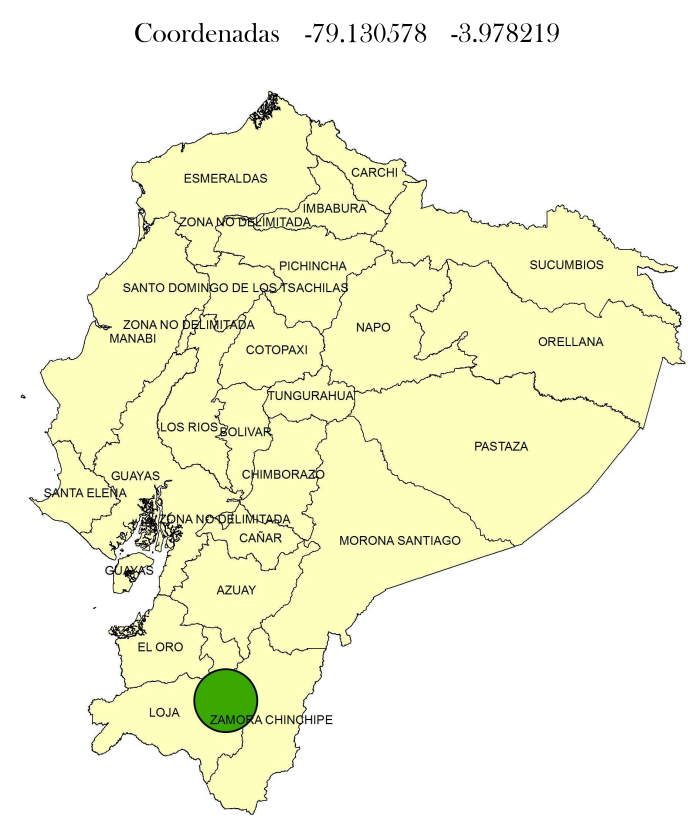

Figura 1: Coordenadas de ubicación Parque Eólico Villonaco.

cuenta con sensores de temperatura, presión atmosférica, veletas y anemómetros; por el momento los datos de temperatura y presión atmosférica no se encuentran disponibles. La velocidad del viento es medida por tres anemómetros de copa, los cuales registran los datos a un nivel temporal de diez minutos para los dos años que se encuentra en funcionamiento la estación automática [3-5]. Por esta razón los datos de la torre meteorológica deben pasar un riguroso estudio de valores atípicos y homogenización de los datos de viento para ser usados en estudios de generación de potencia y energía del Parque Eólico de Villonaco. En la literatura se citan diversos esfuerzos en los estudios de la homogenización y relleno de datos de variables meteorológicas [6-13], de los cuales se hace énfasis en la homogenización de datos de viento citados a continuación [10, 14]. El trabajo realizado por Wan et al. [10] describe la homogenización de las series de viento de 117 estaciones con datos superiores a 47 años y con una temporalidad diaria, lo cual fue usado para la caracterización del clima mediante la velocidad del viento, variabilidad y tendencias [10]. El metadato que indica el tipo de estación, los estándares de instalación y la localización son fundamentales para los procesos de homogenización. Para los valores atípicos, proponen un método de reconocimiento automático bajo los parámetros de módulo de la velocidad del viento $(v)$ superior a $128(\mathrm{~km} / \mathrm{h})$, y si la condición de velocidad al tiempo $(t)$ cumple que, $\left|v_{t}-v_{t-1}\right|>28(\mathrm{~km} / \mathrm{h})$ y $\left|v_{t}-v_{t+1}\right|>28(\mathrm{~km} / \mathrm{h})$ [10]. Este tipo de procedimiento permite establecer una guía al momento de analizar los valores dudosos de una serie temporal obtenida por los registros de una estación automática, con una resolución temporal de diez minutos. La organización Mundial de Meteorología (WMO, por sus siglas en inglés) tiene una serie de recomendaciones al momento de realizar las mediciones para estaciones automáticas, las cuales especifican que los valores pequeños de viento, menores o iguales a $0.2(\mathrm{~m} / \mathrm{s})$, su equivalencia meteorológica es calma, y en general los vientos menores a $1.5(\mathrm{~m} / \mathrm{s})$ son vientos calmados para anemómetros automáticos instalados a 10 metros de altura [15]. Para un parque eólico, las velocidades del viento menores a 3 $(\mathrm{m} / \mathrm{s})$ no son suficientes para mover las paletas de un aerogenerador tipo GoldWind 70/1500, esto según las especificaciones de fábrica [3].

En este trabajo se propone una metodología para el relleno de datos de la torre de medición de Villonaco, debido a que el anemómetro de copa a la altura de 60 metros dejó de funcionar en el mes de octubre de 2013. En este contexto, se realiza un reconocimiento de valores atípicos o dudosos de las series temporales de la velocidad de viento de las distintas alturas de la torre y se analizan dos métodos de relleno de datos.

\section{Métodología y Datos}

El reconocimiento de valores atípicos de las series temporales de velocidad de viento se lo hizo a través del procedimiento de Wan et al. [10] mencionados anteriormente. La homogenización de las series temporales utilizó el trabajo realizado por Goulven et al. [16], el cual propone la homogenización y relleno de datos de las estaciones meteorológicas del Instituto Nacional de Meteorología e Hidrología del Ecuador (INAMHI), mediante el vector regional.

\section{Datos}

Los datos entregados por CELECEP - Unidad de Negocio GENSUR (Corporación Eléctrica del Ecuador Empresa Pública), corresponden a la torre meteorológica automática de Villonaco.

Las mediciones tomadas por la torre de medición de Villonaco correspondientes a la velocidad del viento, se realiza con 3 anemómetros de copa para las alturas de 20,40 y 60 metros.

La velocidad del viento es registrada cada 10 minutos, y se toman los registros del promedio máximo, mínimo y desviación estándar del intervalo de tiempo.

Se realizaron los gráficos de rosa de los vientos para los datos de velocidad del viento y la dirección del viento a 60 metros de altura, y los datos de velocidad y dirección del viento para las altura de 40 y 38 metros respectivamente. Esto se puede apreciar en la Figura 2 y Figura 3.

\section{Metodología}

\section{Valores atípicos y homogenización de datos}

Los valores atípicos se refieren a la identificación de los datos sospechosos medidos por la torre meteorológica, o a su vez, determinar si un sensor de la estación dejó de 


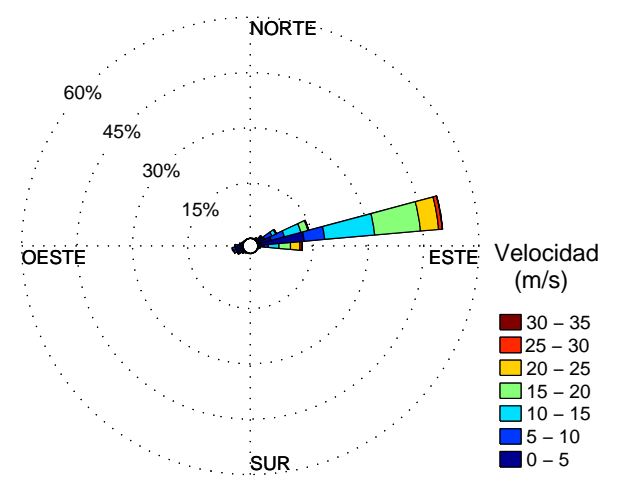

Figura 2: Rosa de los vientos para los datos de velocidad y dirección medidos a 60 metros de altura de la torre meteorológica de Villonaco.

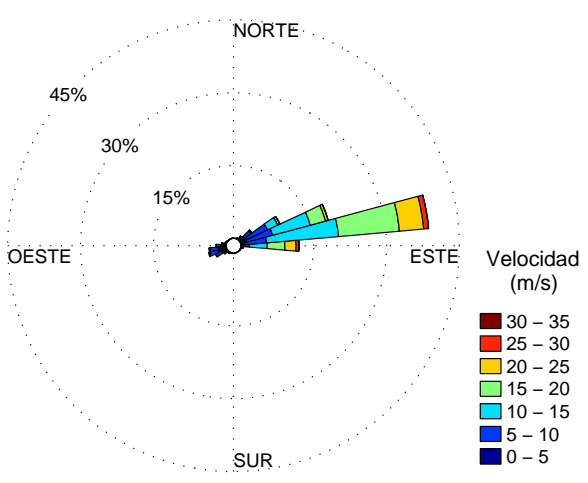

Figura 3: Rosa de los vientos para los datos de velocidad y dirección medidos a 40 y 38 metros de altura de la torre meteorológica de Villonaco.

funcionar o si su calibración cambió. Para esto, la metodología seleccionada consiste en declarar como valores sospechosos si se cumple las siguientes relaciones:

$$
\begin{aligned}
& v_{t}>128(\mathrm{~km} / \mathrm{h}) ; \\
& \left|v_{t}-v_{t-1}\right|<28(\mathrm{~km} / \mathrm{h}) ; \\
& \left|v_{t}-v_{t+1}\right|<28(\mathrm{~km} / \mathrm{h})
\end{aligned}
$$

Donde $v$ es la velocidad del viento.

A partir de las relaciones dadas en la Ecuación (1), se identificaron los valores que cumplen la relación, y en primera instancia, se los reemplazó con el dato -999, para ser tratados y rellenados.

Por otro lado, se propone en este trabajo una identificación de valores atípicos mediante la relación de la velocidad del viento que existe entre las diferentes alturas. Para esto, se busca la correlación existente entre los datos medidos a 20, 40 y 60 metros de altura mediante una comparación a nivel diario, de tal manera que las medias de velocidad quedan definidas de la siguiente manera:

$$
M_{q}=\frac{1}{N} \sum_{i=1}^{N} x_{q}(i), N \in\{\text { datosdeldia }\}, \forall i \in Z_{0}
$$

Donde $M_{q}$ es la media diaria de los datos medidos a 60, 40 y 20 metros de altura.

$$
\begin{array}{r}
R_{6040}=\frac{n \sum_{i=1}^{n} x_{60}(i) x_{40}(i)-\sum_{i=1}^{n} x_{60}(i) \sum_{i=1}^{n} x_{40}(i)}{\sqrt{n \sum_{i=1}^{n} x_{60}(i)^{2}-\left(\sum_{i=1}^{n} x_{60}(i)\right)^{2}}} \times \\
\frac{1}{\sqrt{n \sum_{i=1}^{n} x_{40}(i)^{2}-\left(\sum_{i=1}^{n} x_{40}(i)\right)^{2}}}
\end{array}
$$

En la Ecuación (3) se muestra el coeficiente de correlación entre las mediciones de la torre meteorológica de Villonaco a 60 y 40 metros de altura, esto para la velocidad de viento. Este mismo cálculo se realizó para las opciones de cálculo de los coeficientes de correlación, de tal manera que la matriz de correlaciones queda de la siguiente manera:

$$
R=\left[\begin{array}{ccc}
1 & R_{6040} & R_{6020} \\
R_{4060} & 1 & R_{4020} \\
R_{2060} & R_{2040} & 1
\end{array}\right]
$$

Para identificar si un sensor dejó de funcionar, o si su calibración cambió, se propuso considerar como un dato erróneo si cumple la condición de la Ecuación (5).

$$
\text { dato }_{\text {error }}=\left\{\begin{array}{l}
x_{60}<0,75 x_{40} \\
x_{60}<0,75 x_{20} \\
R_{6040}<0,85 \\
R_{6020}<0,85
\end{array}\right.
$$

Donde, los valores se consideran dudosos si el dato difiere en 0.75 del dato de medición a 40 o 20 metros de altura. Se consideran todas las correlaciones que superen el 0.85 , con lo que se garantiza una buena relación lineal entre dos variables aleatorias. La condición mostrada en la Ecuación (5) se utiliza para la velocidad del viento a 60 metros debido a que a 20 y 40 metros de altura de medición no presentaron errores de medición. En la Figura 4 y la Figura 5 se presentan los gráficos resultantes de las Ecuaciones (2) y (3).

En la Figura 5, se puede observar que para el periodo enero - septiembre de 2013, los valores de correlación de los datos de velocidad de viento varían entre 0.85 y 1. A partir del mes de octubre se nota un cambio de comportamiento en los valores de correlación los cuales varían entre 0 y 0.8 . Estos valores según la metodología 


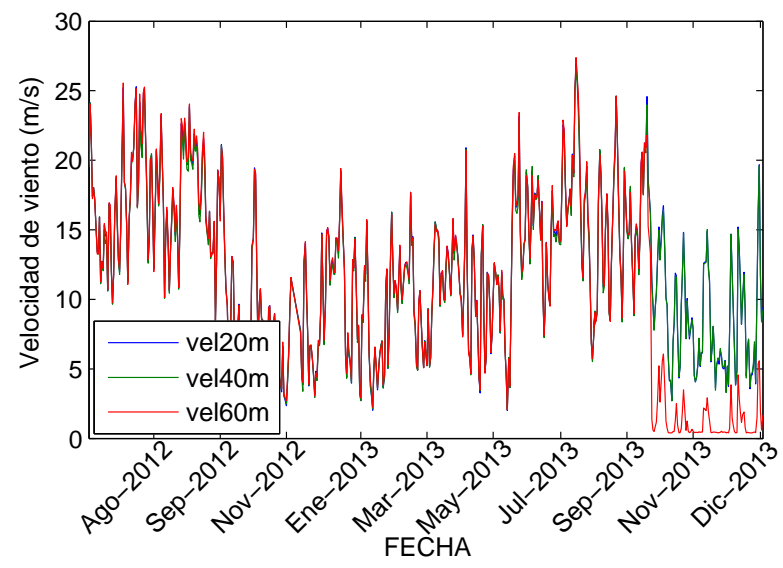

Figura 4: Velocidad del viento en $(\mathrm{m} / \mathrm{s})$ a nivel diario. Se observa que la línea roja cambia su comportamiento a partir de octubre de 2013.

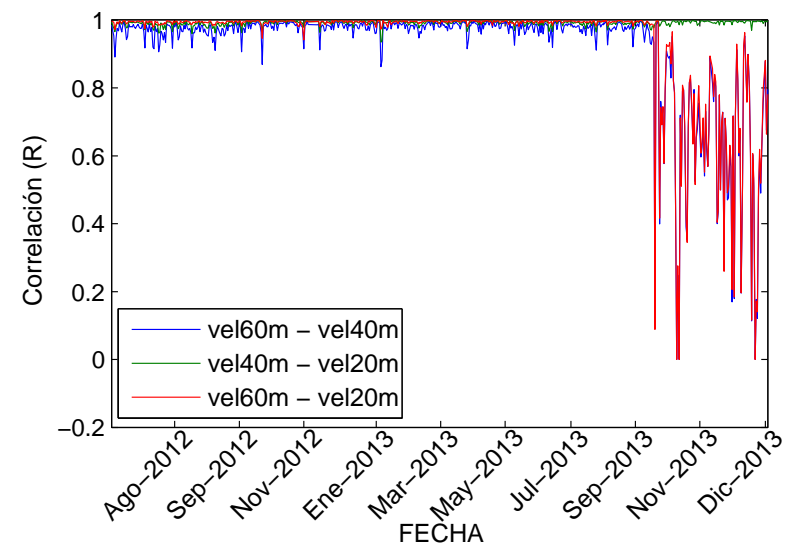

Figura 5: Correlaciones de la velocidad del viento a nivel diario, se observa que las líneas, azul y roja, cambian su comportamiento a partir de octubre de 2013 .

utilizada fueron identificados como mediciones dudosas.

Una vez que se identificaron los valores dudosos de todos los datos de velocidad del viento, se realiza el mismo procedimiento para los valores máximo y mínimo de la velocidad del viento, así como para los registros de desviación estándar de las medidas diezminutales de velocidad.

Al momento de realizar mediciones de velocidad del viento para valores pequeños entre 0 y $1(\mathrm{~m} / \mathrm{s})$ se pueden presentar errores por la precisión del instrumento, el cual para un anemómetro de copa es de $0.22(\mathrm{~m} / \mathrm{s})$. Por esta razón, la WMO propone equivalentes de velocidad del viento, el cual para valores cercanos a $0.2(\mathrm{~m} / \mathrm{s})$ se define como viento en calma, y según la escala de Beaufort son menores a $1(\mathrm{~m} / \mathrm{s})[17,18]$. Para velocidades del viento menores a $3(\mathrm{~m} / \mathrm{s})$ según la escala de Beaufort se define como ventolina. Estos vientos no tienen mayor trascendencia en un parque eólico debido a que no podrían mover las hélices de los aerogeneradores, por lo que en este estudio se procedió a no tomarlos en cuenta en el relleno de datos.

La desviación estándar de la velocidad del viento se en- cuentra relacionada con la intensidad de turbulencia de la siguiente manera:

$$
I T=\frac{\sigma_{v}}{v}
$$

La Ecuación (6) expresa que a mayor dispersión en la medida de la velocidad del viento, la intensidad de turbulencia será mayor. En la Figura 6 se muestra la intensidad de turbulencia vs la velocidad del viento medido a 40 metros de altura.

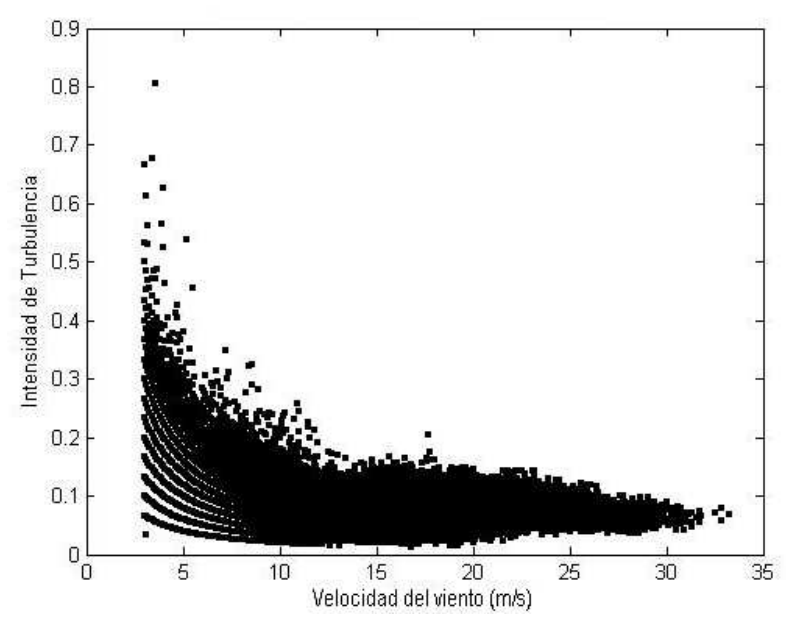

Figura 6: Intensidad de turbulencia vs la velocidad del viento a una altura de medición de $\mathbf{4 0}$ metros de la torre meteorológica de Villonaco.

Como se puede apreciar en la Figura 6, a medida que la velocidad del viento incrementa, la intensidad de turbulencia disminuye. Esto se debe a que la desviación estándar de las mediciones de la velocidad del viento no aumenta o disminuye. Esto es importante para establecer alarmas en un parque eólico.

En este estudio se encontró una relación de decaimiento exponencial entre la intensidad de turbulencia y la velocidad del viento, esta relación se la muestra en la Ecuación (7).

$$
I T(v)=b 1+b 2 \cdot \exp (b 3 \cdot v)
$$

En la Ecuación (7), b1, b2, b3 son constantes, y se determinan mediante una regresión no lineal entre la intensidad de turbulencia $(I T)$ y la velocidad del viento $(v)$. Reemplazando la intensidad de turbulencia de la Ecuación (6) en la Ecuación (7) se puede determinar la desviación estándar de la velocidad del viento.

$$
\sigma_{v}=v(b 1+b 2 \cdot \exp (b 3 \cdot v))
$$

\section{Relleno de datos sospechosos}

El relleno de datos se refiere al reemplazo de los valores dudosos por datos calculados o artificiales que dan un sentido físico a una serie temporal. Por esta razón, es fundamental cumplir con las condiciones mencionadas 
en las Ecuaciones (1) y (5). Una vez encontrados todos los datos sospechosos se proponen dos metodologías de relleno para la velocidad del viento media.

La primera metodología consiste en construir una función multivariada entre las variables de velocidad del viento para las diferentes alturas de medición, tomando en cuenta la restricción de la correlación máxima de la Ecuación (4), considerando la variable a ser rellenada. En nuestro caso se busca rellenar los datos erróneos para la velocidad del viento medido a 60 metros de altura. Para la velocidad del viento a 40 y 20 metros de altura, las mediciones se encuentran estables y pasaron el control de calidad de los datos mencionados en las condiciones de las Ecuaciones (1) y (5).

De esta forma se construye la función de regresión en función de la máxima correlación de los datos de velocidad del viento medidos a 40 o 20 metros de altura. La función de regresión a construirse se muestra en la Ecuación (9).

$$
x_{60}(i)=m x_{q}(i)+b_{q}, i=\{1,2,3, \ldots, N\}
$$

Donde $m$ y $b_{q}$ son las constantes obtenidas en la regresión lineal, $x_{q}$ corresponde a la variable de velocidad del viento cuya correlación es máxima. Debido a que la matriz de correlaciones es una matriz simétrica

$$
\left(R^{T}=R\right) \text {, }
$$

sagduigdteiøxptediómáxima correlación viene dado por la

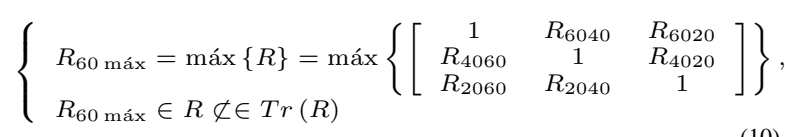

Donde $R_{60 \text { máx }}$ es la correlación máxima entre las variables de velocidad del viento para diferentes alturas de medición y $R_{60 \text { máx }}$ no puede ser igual a los valores de la diagonal principal o los elementos de la traza de $R(\operatorname{Tr}(R))$. Debido a que $R$ es simétrica, y bajo las condiciones de $R_{60 \text { máx }}$, la Ecuación (10) se simplifica de la siguiente manera.

$$
\left\{\begin{array}{l}
R_{60 \text { máx }}=\operatorname{máx}\left\{R_{6040}, R_{6020}\right\} \\
\forall R_{60 i} \in[0,1], i=\{20,40\}
\end{array}\right.
$$

El segundo método viene dado por una regresión múltiple entre las variables de velocidad del viento medidas a 20 y 40 metros de altura. Esta función se encuentra en la Ecuación (12).

$x_{60}(i)=m_{1} x_{40}(i)+m_{2} x_{20}(i)+C_{0}, i=\{1,2,3, \ldots, N\}$

Donde $m_{1}, m_{2}, C_{0}$ son constantes estimadas a partir de mínimos cuadrados o mediante el método de máxima verosimilitud [19].
El relleno para la velocidad del viento diezminutal máxima y mínima tiene la misma metodología que se expuso anteriormente para la velocidad media. Para el relleno de la desviación estándar se utilizó la relación que se encontró en la Ecuación (8), debido a que la intensidad de turbulencia y la velocidad del viento tienen un comportamiento de la forma de un decaimiento exponencial.

Los datos atípicos reconocidos por las Ecuaciones (1) y (5) no son aptos para la desviación estándar de la velocidad del viento diezminutal, por este motivo se consideraron como valores dudosos de la desviación de estándar, o de la intensidad de turbulencia, a los valores que no pasaron el control de calidad mencionados en las Ecuaciones (1) y (5).

\section{Resultados y Discusión}

En esta sección se presentan los resultados de homogenización y relleno de las observaciones de viento de la torre meteorológica de Villonaco.

Con la metodología propuesta se logró identificar el o los anemómetros que tuvieron valores dudosos. En este contexto, el anemómetro instalado a 60 metros de altura dejó de funcionar el 24 de septiembre de 2013.

El número de días que el anemómetro dejó de registrar datos son 168 , ya sean por fallas o por la calibración del instrumento. Los datos correspondientes a estos días se encontraron como dudosos debido a la pobre relación y por la cantidad de datos atípicos que se registraron al momento de realizar el control de calidad.

La metodología de relleno permitió crear los datos de velocidad del viento media, máxima, mínima y desviación estándar. El resultado de los dos métodos propuestos se muestra en la Figura 7 y Figura 8.

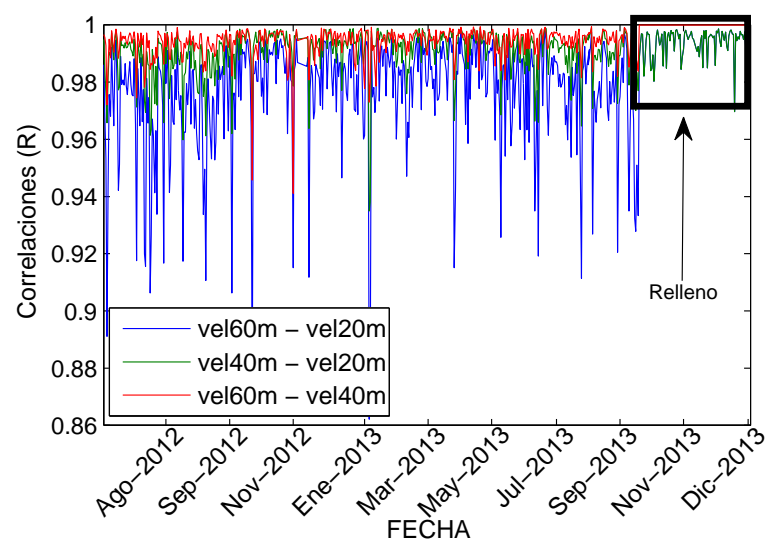

Figura 7: Correlación de los datos rellenados a partir del primer método para la velocidad del viento medida a 60 metros de altura.

Es posible observar en la Figura 7 que los coeficientes de correlación correspondientes a las velocidades de viento relleno a 60 metros, en relación a los 40 metros 
de altura medición son igual a 1. Esto se debe a que el primer método escoge la máxima correlación entre las variables analizadas mediante la Ecuación (11). Los coeficientes de correlación entre las variables de velocidad de viento a 60 y 20 metros de altura convergen a los coeficientes de correlación de las variables observadas a 40 y 20 metros, debido a la relación que tienen las variables de velocidad a 60 metros con las de 40 metros.

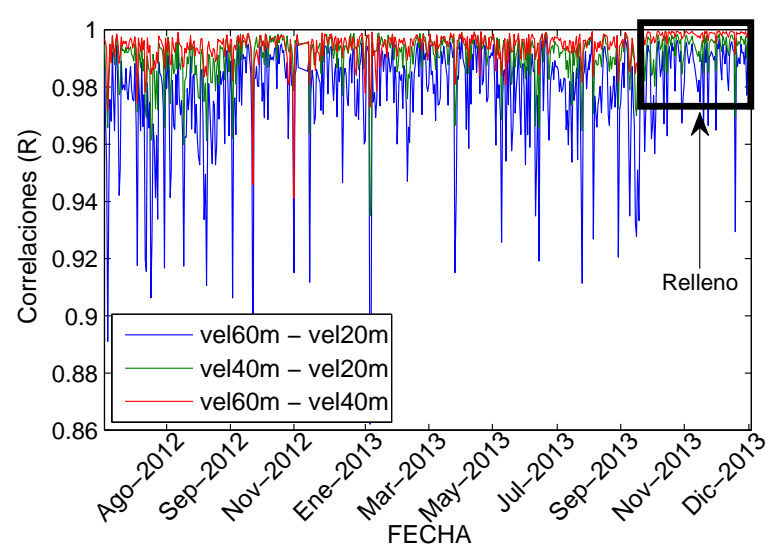

Figura 8: Correlación de los datos rellenados a partir del segundo método para la velocidad del viento medida a 60 metros de altura.

Como se puede apreciar en la Figura 8, la velocidad del viento relleno a 60 metros conserva un comportamiento similar a las observaciones de velocidad medidas a 40 y 20 metros de altura. Los coeficientes de correlación son cercanos a uno, lo cual nos indica una relación muy buena entre todas las variables. A diferencia del primer método, el segundo método optimiza la relación entre las variables medidas a 20 y 40 metros, pero la correlación entre las mediciones a 60 y 40 metros de altura es la más alta. Esto se debe a que la correlación de los observables considerados para construir la función de multi-regresión es mayor con el observable medido a 40 metros, como fue demostrado en el primer método.

La comparación de ambos métodos se lo realiza a partir del gráfico de correlaciones y velocidad del viento, rellenados para ambas variables. En primera instancia, se muestra en la Figura 9 la comparación de los rellenos de velocidad del viento media para la altura de medición de 60 metros. Es posible observar que el relleno por el primer método supone que la velocidad del viento a 60 metros siempre es mayor a la de las mediciones a 40 metros. Esto no sucede siempre debido a que se presentan ráfagas que pueden alterar ese comportamiento, o a su vez, por la turbulencia debido a torbellinos o velocidad en el eje z del vector viento. Para el segundo método, este comportamiento no es un problema debido a que considera el comportamiento de las series de temporales de las mediciones de la velocidad a 20 y 40 metros de altura.

En la Figura 10 se puede observar el gráfico de dispersión entre los datos relleno mediante los dos métodos
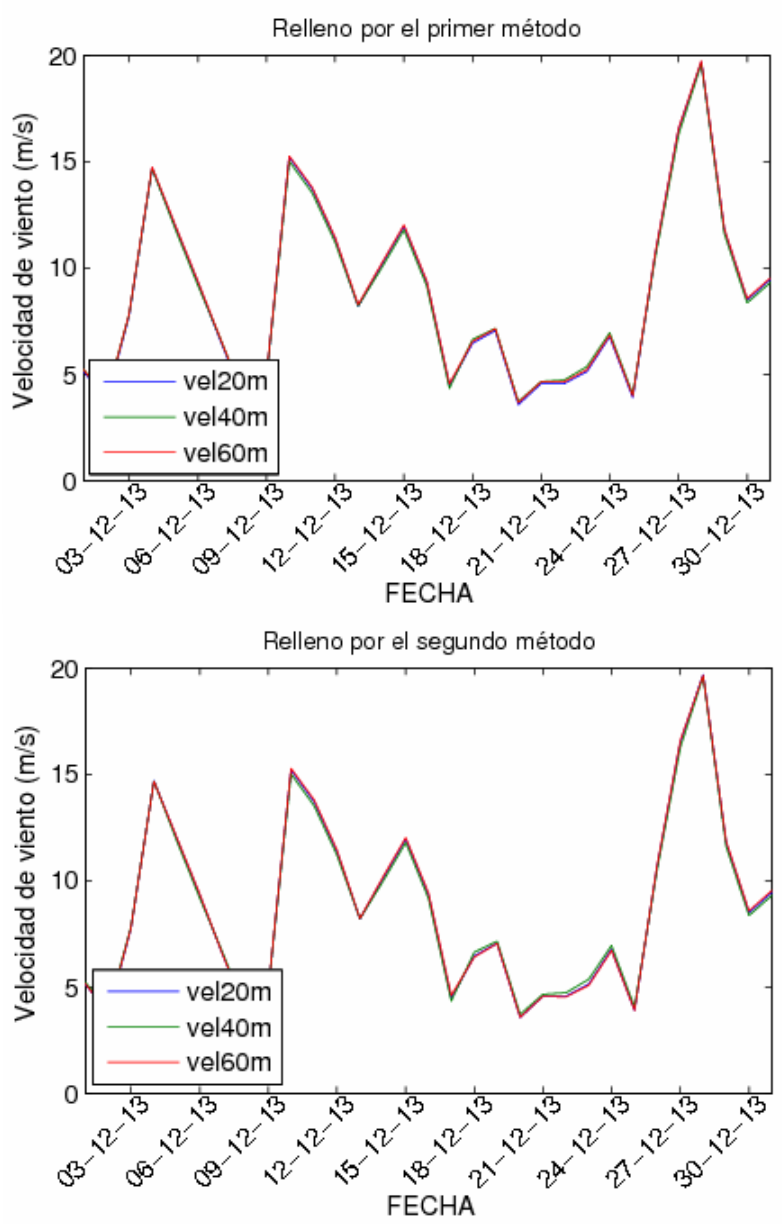

Figura 9: Comparación de los datos relleno de velocidad del viento para las diferentes alturas de medición. En el gráfico superior se presenta el relleno por el primer método, el inferior corresponde al relleno por el segundo método.

explicados en la metodología. Para los valores de velocidad del viento cercanos a cero es posible observar una dispersión mayor que para los datos para la velocidad mayor a $20(\mathrm{~m} / \mathrm{s})$. Los dos métodos de relleno tienen una correlación de 0.9994 , lo cual nos indica que su comportamiento es muy similar. Las medias para los dos rellenos se muestran a continuación.

$$
\begin{aligned}
& \bar{v}_{O P C I O N 1}=8,7(\mathrm{~m} / \mathrm{s}) \\
& \bar{v}_{O P C I O N 2}=8,6(\mathrm{~m} / \mathrm{s}) ;
\end{aligned}
$$

Es fácil apreciar que los dos métodos se asemejan, tanto en su media como en su relación. La diferencia entre ambos es la suposición que se mencionó anteriormente, la cual implica que el relleno a 60 metros siempre debe ser mayor a la velocidad del viento a 40 metros, y esto no siempre sucede.

Una vez realizado el relleno para la velocidad del viento, se procedió el tratamiento de datos de velocidad del viento máxima y mínima diezminutal. Los procedimientos fueron los mismos, pero el comportamiento para la velocidad del viento mínimo obtuvo unos coeficientes de correlación mucho más bajos que los obtenidos para 


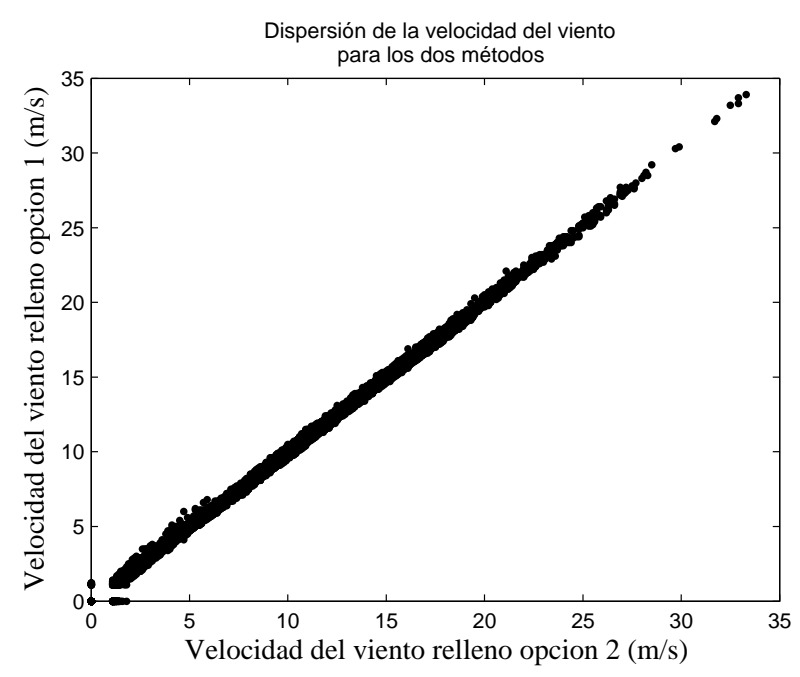

Figura 10: Gráfico de dispersión para los rellenos de con los dos métodos.

las comparaciones entre las velocidad del viento máxima y media.

Una vez escogido el método de relleno para la velocidad del viento, se procede a completar los datos de la desviación estándar mediante la intensidad de turbulencia.

Para el relleno de la desviación estándar de la velocidad del viento, se procede a calcular los coeficientes de la Ecuación (7), con la restricción de la velocidad del viento siempre mayor o igual a $3(\mathrm{~m} / \mathrm{s})$, debido a las restricciones de viento para el funcionamiento de aerogeneradores. Por otro lado, se rellenaron los valores de desviación estándar con la Ecuación (8) a partir de la estimación de las constantes en los intervalos de velocidad del viento para $[1,2[(\mathrm{~m} / \mathrm{s})$ y $[2,3[(\mathrm{~m} / \mathrm{s})$. Esto se lo hizo por la relación de la intensidad de turbulencia con la velocidad del viento, la cual aumenta considerablemente al disminuir la velocidad. Todos los valores de velocidad del viento menor a $1(\mathrm{~m} / \mathrm{s})$ se consideraron como cero, así como su desviación estándar, ya que se los considera como viento en calma y ventolinas, y por tanto no tienen gran trascendencia en el parque eólico.

Los coeficientes de la Ecuación (8), a partir de la regresión mostrada en la Figura 11, quedan de la siguiente manera.

$$
\sigma_{v}=v(0,06+0,30 \exp (-0,38 v)), \forall v \geq 3(\mathrm{~m} / \mathrm{s})
$$

A partir de la Ecuación (14) se procedió a realizar el relleno para la variable de la desviación estándar de la velocidad del viento, para los datos de velocidad mayores o igual a $3(\mathrm{~m} / \mathrm{s})$. Las ecuaciones para las restricciones de velocidad del viento $[1,2[(\mathrm{~m} / \mathrm{s})$ y $[2,3[(\mathrm{~m} / \mathrm{s})$ se muestran a continuación.

$$
\begin{aligned}
& \sigma_{v}=v(0,06+0,78 \exp (-0,65 v)), \forall v \in[1,2[(\mathrm{~m} / \mathrm{s}) \\
& \sigma_{v}=v(0,06+0,40 \exp (-0,44 v)), \forall v \in[2,3[(\mathrm{~m} / \mathrm{s})
\end{aligned}
$$

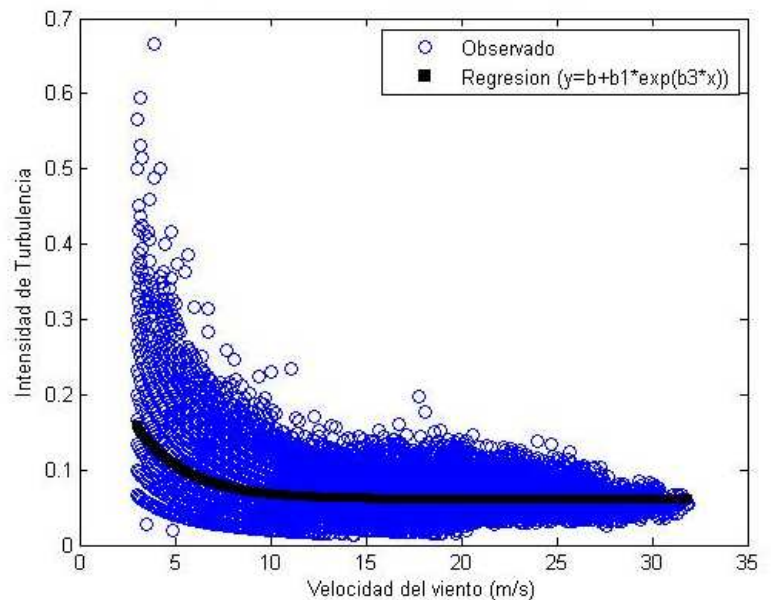

Figura 11: Intensidad de turbulencia estimada a partir de las Ecuaciones (6) y (7).

La Ecuación (15) es utilizada para el relleno de la desviación estándar de la velocidad del viento, la intensidad de turbulencia aumenta cuando la velocidad del viento es pequeña. La desviación estándar de la velocidad es cero para valores menores a $1(\mathrm{~m} / \mathrm{s})$.

Al final se construyó una base de datos tipo Access, usando el control de conectividad libre OBDC de Windows, la cual consiste en los valores analizados por los procedimientos mencionados en este artículo. Por último, se presenta el gráfico de la rosa de los vientos de la base de datos relleno para todos los datos diezminutales observados y rellenados a 60 metros de altura de medición (Figura 12).

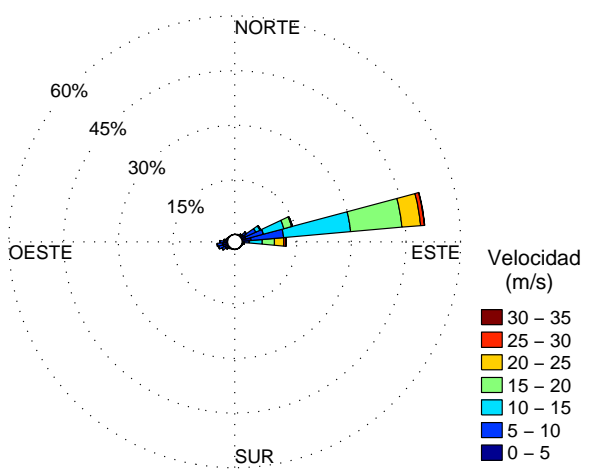

Figura 12: Rosa de los vientos para la variable de velocidad del viento medida a 60 metros de altura.

\section{Conclusiones y Recomendaciones}

En este estudio se realizó satisfactoriamente un análisis de valores atípicos que permite identificar todas las mediciones de velocidad del viento consideradas como valores dudosos. Se establecieron parámetros para un control de calidad de los datos que permiten dar un sentido real a una serie temporal de velocidad del viento. 
Los métodos de relleno para la velocidad del viento media, máxima y mínima son muy similares en su comportamiento como serie de datos, los valores medios no se diferencian en más del $0.01(\mathrm{~m} / \mathrm{s})$. La suposición del primer método, es decir que la velocidad del viento a 60 metros siempre debe ser mayor a la medida en 40 metros, se pudo comprobar que no siempre es correcta. Por este motivo se escogió el segundo método como procedimiento para rellenar datos dudosos.

Por último, la desviación estándar mediante la ecuación empírica de la intensidad de turbulencia, demostró un comportamiento de decaimiento exponencial. Un dato interesante es el cambio de las constantes de la curva de decaimiento exponencial para los diferentes intervalos de velocidad analizados, los cuales para velocidades del viento pequeñas, las constantes de decaimiento exponencial aumentan.

Una base de datos con el análisis de este trabajo fue creada en Access, la cual contiene el metadato de las variables de la torre meteorológica de Villonaco. Esto ayudará a las simulaciones de producción energética del parque eólico al proyecto de "Análisis del comportamiento de un parque eólico en condiciones extremas" desarrollado por INER.

El siguiente paso será el de homogenizar los datos de viento de cada uno de los aerogeneradores con el estándar de la torre meteorológica de Villonaco, de esta manera el análisis del comportamiento de las turbinas, referente al rendimiento, producción y alarmas, no se verá afectado por valores perdidos o anómalos.

\section{Agradecimientos}

Un agradecimiento muy especial para el grupo de eólica del Instituto Nacional de Eficiencia Energética y Energías Renovables. A la Corporación Eléctrica del Ecuador (CELEC-EP) por facilitar toda la información meteorológica. A la Secretaría de Educación Superior, Ciencia, Tecnología e Innovación por financiar este proyecto

\section{Referencias}

[1] Corporación Eléctrica del Ecuador (CELEC-EP) y Ministerio de Electricidad y Energía Renovable (MEER). 2012. "Parque Eólico Villonaco": 3-34. https : //www. celec.gob.ec/index.php?option=com_content\& view=article\&id=189: eolico.

[2] Xinjiang Goldwind Science and Tefchnology Co. Ltda China. 2011. "Goldwind 1.5MW Permanent Magnet Direct-Drive (PMDD) Wind Turbine”. 1: 1-6.

[3] Coppin, P. A; Taylor, K. J. 1983. "A three-component sonic anemometer/thermometer system for general micrometeorological research". Boundary-Layer Meteorology. 27(1): 27-42. (10.1007/BF00119970)
[4] Wilczak, J. M.; Steven, P. Oncley and Stage, S. A. 2000. "Sonic Anemometer Tilt Correction Algorithms". Boundary-Layer Meteorology. 99(1): 127-150. (10.1023/A:1018966204465)

[5] Yufeng, Z.; Yan, W. 2011. "To Measure Wind Speed using the theory of One-dimensional Ultrasonic Anemometer". Bachelor's Thesis in Electronics, University of G. ${ }^{a}$ vle: $1-40$

[6] Boulanger, P. P.; Aizpuru, J.; Leggieri, L.; Marino, M. 2010. "A procedure for automated quality control and homogenization of historical daily temperature and precipitation data $(\mathrm{APACH})$ : part 1: quality control and application to the Argentine weather service stations". Climatic Change. 98: 471-491. (10.1007/s10584-0099741-9)

[7] Štěpánek, P.; Zahradníček, P.; Farda, A. 2013. "Experiences with data quality control and homogenization of daily records of various meteorological elements in the Czech Republic in the period 1961Ú2010". Quarterly Journal of the Hungarian Meteorological Service. 117(1): 123-141.

[8] Aguilar, E.; Auer, I; Brunet, M.; Peterson, T. C.; Wiering, J. 2003 " Guidelines on Climate Metadata and Homogenization”. World Meteorological Organization, (1186): $1-50$.

[9] Domonkos, P. 2011. "Homogenising time series: beliefs, dogmas and facts". Adv. Sci. Res. 6: 167Ü172. (10.5194/asr-6-167-2011)

[10] Wan, H.; Wang, X. L.; Swail, V. R. 2010. "Homogenization and Trend Analysis of Canadian Near-Surface Wind Speeds". Journal of Climate / American Meteorological Society. 23: 1209-1225. (10.1175/2009JCLI3200.1)

[11] Menne, M. J.; Williams Jr., C. N. 2009 "Homogenization of Temperature Series via Pairwise Comparisons". Journal of Climate / American Meteorological Society. 22: 1700-1717. (10.1175/2008JCLI2263.1)

[12] Haimberger, L. 2007, "Homogenization of Radiosonde Temperature Time Series Using Innovation Statistics". American Meteorological Society. 20: 1377-1403. (10.1175/JCLI4050.1)

[13] Kuglitsch, F. G.; Toreti, A.; Xoplaki, E.; Della-Marta, P. M.; Luterbacher, J.; Wanner, H. 2009. "Homogenization of daily maximum temperature series in the Mediterranean". Journal of Geophysical Research. 114: 1-16. (10.1029/2008JD011606)

[14] Thomas, B. R.; Kent, E. C.; Swail, V. R. 2005, "Methods to Homogenize Wind Speeds from Ships and Buoys". Int. J. Climatol 25: 979Ú'995. (10.1002/joc.1176)

[15] WMO. 2008. "Guide to Meteorological Instruments and Methods of Observation / Measurement of Surface Wind". CIMO-Guide, 1: 5-14.

[16] Goulven, p.; Aleman, M. A.; Osorno, I. 1988. "Homogenización y Regionalización Pluviométrica por el Método del Vector Regional". V Congreso de Hidráulica - AEHID/CICP: 1-118. https://www . wmo.int/ pages/prog/www/IMOP/CIMO-Guide.html. 
[17] WMO. 2012. "Manual on Marine Meteorological Services". Annex VI to WMO Technical Regulafions. I(5 ) http://library.wmo.int/pmb_ged/ wmo_558_en-v1.pdf.

[18] Met Office. 2011. "The Beaufort Scale". National Meteorological Library and Archive Fact sheet $\mathrm{I}(6)$ www. metoffice.gov.uk/corporate/library

[19] Peña, D. 2002. "Análisis de Datos Multivariantes", McGraw-Hill Interamericana de España: España. 